\title{
A High Speed Coprocessor for Elliptic Curve Scalar Multiplications over $\mathbb{F}_{p}$
}

\author{
Nicolas Guillermin ${ }^{1,2}$ \\ 1 DGA Information Superiority, Bruz, France \\ 2 IRMAR, Université Rennes 1, France
}

\begin{abstract}
We present a new hardware architecture to compute scalar multiplications in the group of rational points of elliptic curves defined over a prime field. We have made an implementation on Altera FPGA family for some elliptic curves defined over randomly chosen ground fields offering classic cryptographic security level. Our implementations show that our architecture is the fastest among the public designs to compute scalar multiplication for elliptic curves defined over a general prime ground field. Our design is based upon the Residue Number System, guaranteeing carry-free arithmetic and easy parallelism. It is SPA resistant and DPA capable.
\end{abstract}

Keywords: elliptic curve, high speed, RNS, prime field, FPGA.

\section{Introduction}

Twenty five years after their introduction for cryptographic applications [14, elliptic curves are well established in the field of public key cryptography. A standard of the National Institute of technology (NIST) recommends their use for digital signature [17]. The most time consuming operation in elliptic curve based protocols is the scalar multiplication. As a consequence, scalar multiplication has attracted a lot of attention in public literature. Available designs may differ greatly depending on the target implementation (GPGPUs, CPUs, ASICs, FPGAs) and the aim they try to achieve which may be related to speed, size, power consumption or security issues. We refer the reader to 3251021 for example of known implementations.

In this paper, we describe the fastest available architecture for computing $[k] G$ over curves defined over $\mathbb{F}_{p}$ for general prime $p$ in FPGA. Our architecture is based on Residue Number Systems (RNS) and is resistant against side channel attacks. We have made a FPGA implementation of our design. Actually, FPGA implementations are particularly interesting for at least two reasons : they are well suited to provide a good local protection level, and they constitute generally the first step towards faster ASIC implementations.

Target application of such special purpose designs are all the fields where both high speed, low latency and high level resistance against attacks are required (example : IPSEC set-top box). 
Related work : A great overview of high speed hardware accelerator for ECC is given by [13. Designs can be split in two categories : those which support elliptic curves over $\mathbb{F}_{2^{n}}$, and those over $\mathbb{F}_{p}$. Architectures of the first group give the best speed to security ratio. It is mostly due to the field structure (No carry is propagated). State of the art implementations show a latency under $20 \mu$ s for a $2^{80}$ security [8]. Nevertheless large characteristic remain interesting, mostly because $\mathbb{F}_{p}$ offers less structure than $\mathbb{F}_{2^{n}}$, and may be safer. Some architecture can also support both field types 22 .

Some implementations are specific to a pseudo-Mersenne prime [7. These implementations may be faster than the one which do not depend on the relying field. Nevertheless the ability of changing the curve is also an asset for security (finding weak curves is still an active research area). Our architecture is of this kind. To our knowledge the best architecture is the one of Mentens [11, which computes a 1 ms 160 bit scalar multiplication on a Xilinx Virtex 2 pro. Most of the implementations are based on a multi-precision Montgomery representations of numbers, allowing reduction without expensive divisions.

Another way to represent big numbers is the Residue Number System. It provides fast and carry free arithmetic. A modified version of the Montgomery algorithm [1] makes it suitable for arithmetic in $\mathbb{F}_{p}$. Szerwinski et al [25] used it to produce the fastest software implementation of scalar multiplication. Kawamura et al [1016] proposed a very efficient architecture for RSA signature on an ASIC. Their contribution is analysed in section 3, since it is the starting point of our work.

The higher $p$ is, the more efficient RNS is (because its high parallelization ability). Then we could think that applying RNS to ECC will be less interesting than RSA. We show in this paper that this drawback is compensated by 2 advantages. First, the RNS ability to execute patterns like $A B+C D$ in only one reduction, while both products are almost free, reduces the time of point operations in ECC while it is useless for RSA (2.2). Second, ECC operations are parallelizable, therefore we can deepen the pipelines while keeping a high rate occupation (3.2).

Our contribution : We present a complete redefinition of main module of Kawamura. Thanks to it we can use elliptic curve and we reach high speed on a FPGA. We design the first architecture to break $1 \mathrm{~ms}$ for 160 bit elliptic curve scalar multiplication over prime field of any characteristic, even on a $130 \mathrm{~nm}$ node FPGA (the Altera Stratix family in our case). Our scalable architecture keeps its advantage even for larger groups (up to 512 bits). We also propose an algorithm for RNS-Radix transformation that does not cost a single gate, and base choice considerations for RNS use with elliptic curves.

Structure of the paper : The section 2 deals with mathematical backgrounds of RNS and elliptic curves. The section 3 describes and analyses the choices that are made to improve Kawamura's architecture, and and the section 4 gives the results of implementations, and compares it to other existing design. Design schemes are at the end of the paper. 


\section{Mathematical Background}

Notations : In all the paper, for $a, b \in \mathbb{N}$, we denote by $|a|_{b}$ the result of $a$ modulo $b$.

\section{$2.1 \quad$ RNS}

Overview : Let $B=\left\{m_{1}, \cdots, m_{n}\right\}$ be a set of co-prime natural integers, and $M=\prod_{i=1}^{n} m_{i}$. The residue number system (RNS) representation $\{X\}_{B}$ of $X \in$ $\mathbb{N}$ such that $0 \leq X<M$ is the unique set of positive integers $\left\{x_{1}, \cdots, x_{n}\right\}$ with $x_{i}=|X|_{m_{i}}$. This representation allows fast arithmetic in $\mathbb{Z} / M \mathbb{Z}$ since

$$
\{X \odot Y\}_{B}=\left\{\left|x_{1} \odot y_{1}\right|_{m_{1}}, \cdots,\left|x_{n} \odot y_{n}\right|_{m_{n}}\right\}
$$

for $\odot \in(+,-, \times, /), /$ being only available for $Y$ coprime with $M$. The integer $X$ is recovered thanks to the Chinese remainder theorem :

$$
X=\left|\sum_{i=1}^{n}\right| x_{i} \times\left. M_{i}^{-1}\right|_{m_{i}} \times\left. M_{i}\right|_{M} \text { where } M_{i}=\frac{M}{m_{i}} .
$$

Note that $M_{i}^{-1}$ is then well defined in $\mathbb{Z} / m_{i} \mathbb{Z}$. In the rest of the paper, $B$ is called a RNS base and the $\left\{m_{i}\right\}_{i=1, \ldots, n}$ are called channels of $B$, since every calculation are done independently modulo these channels.

RNS Montgomery reduction algorithm : The Montgomery reduction application was first introduced in [15] for the purpose of multiprecision arithmetic. The paper [1] presents an adaptation in the context of RNS representation.

In the following we recall the main results of this last paper. Let $p$ be a prime, $\alpha>2$ an integer, $B$ and $\tilde{B}$ be two RNS bases with their channel products $M$ and $\tilde{M}$ such that $M>\alpha p$ and $\tilde{M}>2 p$. For all input $a<\alpha p^{2}$ given in $B$ and $\tilde{B}$ the Montgomery algorithm computes $S$ in $B$ and $\tilde{B}$ such that $S<2 p$ and $|S|_{p}=\left|a \times M^{-1}\right|_{p}$. The factor $M^{-1}$ is not a concern if a lot of computation in modular arithmetic are to be done in a row, which is the case in most applications related to cryptography. Actually, thanks to the use of Montgomery representative $\phi(X)=|X M|_{p}$ (see [15]), one only needs a transformation at the beginning of any calculation $\phi(X)=\operatorname{Re}_{\text {Montg }}\left(X \times\left|M^{2}\right|_{p}, p, B, \tilde{B}\right)$ and the corresponding invert transformation $\phi^{-1}(Y)=\operatorname{Red}_{\text {Montg }}(Y, p, B, \tilde{B})$ at the end.

The base change $B_{\text {ext }}(X, B, \tilde{B})$ is due to the fact that one can not divide by $M$ in $B$. The second base extension computes $\{S\}_{B}$ from $\{S\}_{\tilde{B}}$. Both are then available for another computation. Unlike the multiprecision Montgomery algorithm, we can not execute the final reduction, since it is not easy to know if $S$ is more or less than $p$ (comparison is a greedy operation in RNS representation). The result $S$ is then kept between 0 and $2 p$. The main consequence is that $\tilde{M}$ has to be up to $2 p$. The choice of $B$ depends on the maximal number we wish to reduce. Proposition 1 shows that $\tilde{M}$ does not need to be more than $M$. Even if $\tilde{Q}$ is not equal to $Q$ but to $|Q|_{\tilde{M}}$, the algorithm still gives the correct output. 


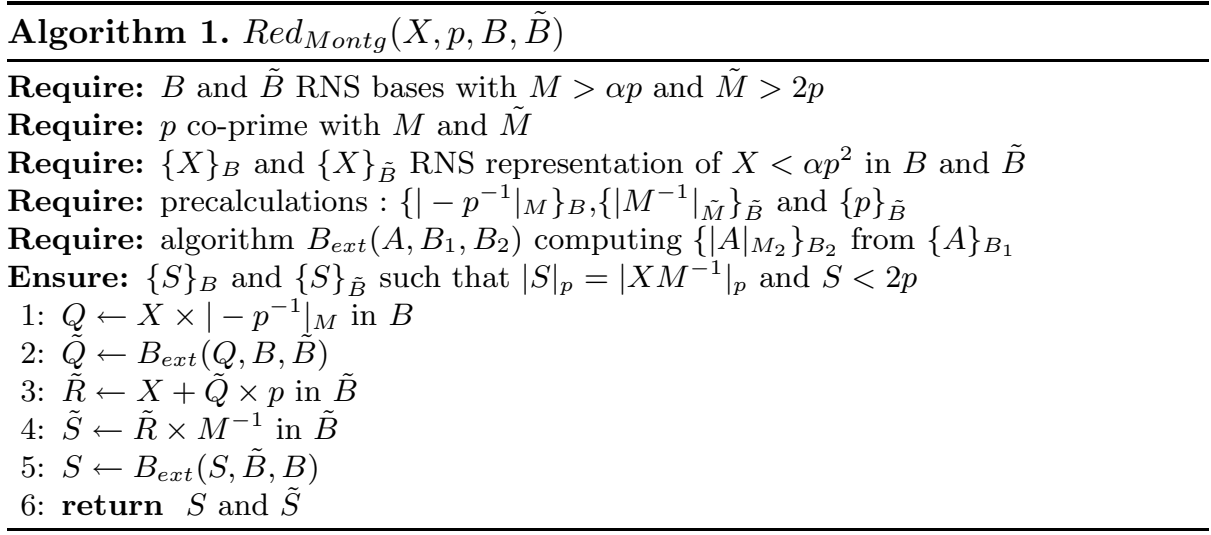

Proposition 1. Given $\alpha>2$, if $M>\alpha p$ and $\tilde{M}>2 p$ then $\operatorname{Red}_{M o n t g}(X, p, B, \tilde{B})$ gives the correct output for every $X$ between 0 and $\alpha p^{2}$.

Proof. Be $X<\alpha p^{2}, M>\alpha p$ and $\tilde{M}>2 p . Q=\left|X P^{-1}\right|_{M}$, therefore $Q<M$. By $B_{\text {ext }}, \tilde{Q}=|Q|_{\tilde{M}}$. As $X_{\tilde{B}}=|X|_{\tilde{M}}$ and $p<\tilde{M}, \tilde{S}$ is equal to $|(X+Q p) / M|_{\tilde{M}}$. As $Q<M, T=(X+Q p) / M<2 p$. Therefore $\tilde{S}=T$, and as $\alpha>2 S=T$ too. As $T \equiv\left|X M^{-1}\right|_{p}$, we can conclude that $S$ and $\tilde{S}$ are the expected results.

RNS base extension : The greediest steps of this algorithm are the two base extensions $B_{\text {ext }}(X, B, \tilde{B})$ and $B_{\text {ext }}(X, \tilde{B}, B)$. They are a classical $O\left(n^{2}\right)$ algorithm, where $n$ is the RNS base size, and the elementary operation is a modular multiplication/addition on a channel. The main concern of every algorithm implementing $B_{\text {ext }}$ is to provide a way to compute the final reduction by $M$, to calculate $\gamma$ such that

$$
X=\sum_{i=1}^{n}\left|x_{i} \times M_{i}^{-1}\right|_{m_{i}} \times M_{i}-\gamma M .
$$

Once $\gamma$ is calculated, $X$ is easily recovered on every channel $\tilde{m}_{i}$ by multiplying and accumulating the result. Three different algorithm are proposed by literature :

- a Mixed Radix System (MRS) approach 2] which natively avoids final reduction, but is hard to implement in hardware because of the structure of the algorithm, but remains a good alternative in software,

- an extra modulus approach proposed by Shenoy and Kumaresan 24]. The idea of this algorithm is to have a $m_{e}$ coprime with $M$ and $\tilde{M}$, and to use $X\left[m_{e}\right]$ to compute $\gamma$. The main drawback of this approach is that we need to keep $|X|_{m_{e}}$ all along during the calculation, while we just need it during the base extension,

- a floating point approach proposed by Posch and Posch [19], and improved by Kawamura et al. 10. The main idea is to transform the equation 3 as follow:

$$
X=\sum_{i=1}^{n} \frac{M \xi_{i}}{m_{i}}-M\left\lfloor\sum_{i=1}^{n} \frac{\xi_{i}}{m_{i}}\right\rfloor \text { with } \xi_{i}=\left|x_{i} M_{i}^{-1}\right|_{m_{i}}
$$




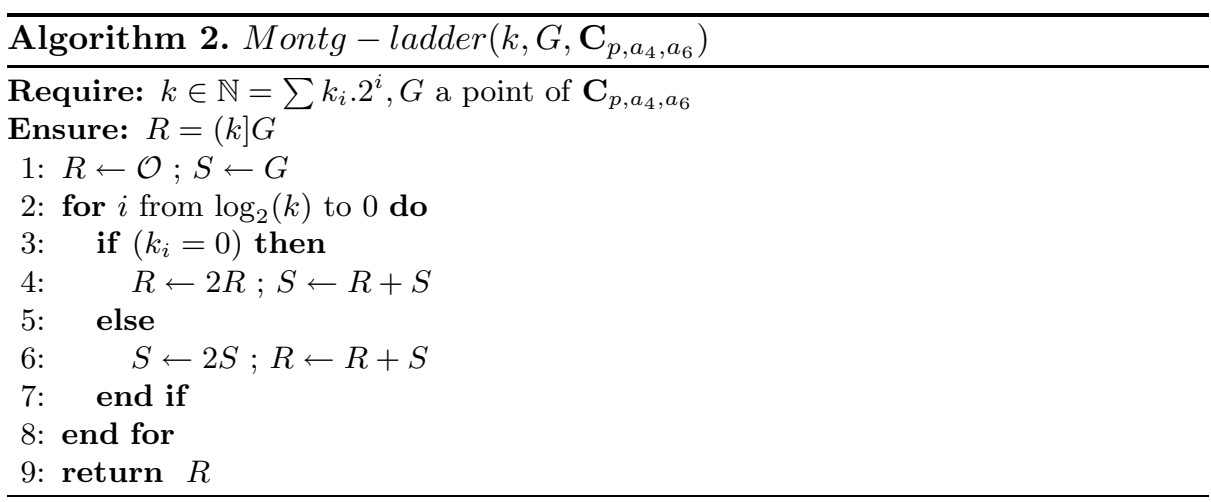

The main drawback of the floating point approach is a potential emergence of an offset due to the approximation while computing $\left\lfloor\sum_{i=1}^{n} \xi_{i} / m_{i}\right\rfloor$. In [10] $\xi_{i} / m_{i}$ is approximated by $\xi_{i} / 2^{r}$ where $r$ is chosen as word depth in the proposed architecture. As $m_{i}$ is chosen as a pseudo-Mersenne prime near $2^{r}$, the offset of the calculation may be easily limited to $1 / 2$. In [10] is explained how this possible error can be without consequences for the result, as soon as $\tilde{M}<6 p$. The output of $\operatorname{Red}_{M o n t g}$ will be less than $3 p$, whatever the input is (proposition 1 is then easily adapted, with $\tilde{M}>6 p$ and $\alpha>3$ the output of the algorithm 1 will be less than $3 p$ ).

\subsection{Elliptic Curves}

Overview : In this paper, considered elliptic curves $\mathbf{C}_{p, a_{4}, a_{6}}$ are seen as sets of couples $(x, y) \in \mathbb{F}_{p}^{2}$ verifying the following equation, with $p$ prime and extra conditions on $a_{4}$ and $a_{6}$ which are not discussed here.

$$
y^{2}=x^{3}+a_{4} x+a_{6}
$$

Together with the point at infinity $\mathcal{O}, \mathbf{C}_{p, a_{4}, a_{6}}$ is an abelian group. The composition law has a geometric meaning described by the vertical and tangent. Some specific curve shapes (forms of the equation) spare multiplications and reduction while computing $P+Q$ and $2 P$ over $\mathbf{C}_{p, a_{4}, a_{6}}$ 95].Nevertheless the Weierstrass form represents all elliptic curves over prime fields (through isomorphism over $\mathbb{F}_{p}^{2}$ ). Other representations can only represent curves with subgroups (e.g order 4 for Edwards curves and Montgomery form, order 3 for Hessian curves...). Here we consider general curves in Weierstrass form, given by 5 .

Addition and doubling formulæ : The Montgomery ladder [9] algorithm is a SPA-resistant square and multiply algorithm, computing $[k] G$ over $\mathbf{C}_{p, a_{4}, a_{6}}$ using one double and one add per bit of $k$.

Moreover, one can use projective coordinates $X_{P}, Y_{P}, Z_{P}$ of point $P=$ $x_{P}, y_{P}$ where $x_{P}=X_{P} / Z_{P}$ and $y_{P}=Y_{P} / Z_{P}$ when $Z_{P} \neq 0$. With projective coordinates every point of the curve has $p-1$ different representation. This can be used to execute leak-resistant computation (by changing the point representation before realizing the scalar multiplication) 44. Point additions and doubling are then computed without inversion in $\mathbb{F}_{p}$. 
Combined with algorithm 2, we can spare the computation of $Y_{[k] G}\left(y_{[k] G}\right.$ is recomputed at the end of the algorithm if necessary). Formulæ for adding and doubling points optimized for RNS are given in [20]. We briefly recall them in the following table.

\begin{tabular}{|c|c|}
\hline$P+Q$ & $2 P$ \\
\hline$A \leftarrow Z_{P} X_{Q}+X_{P} Z_{Q}$ & $E \leftarrow Z_{p}^{2}$ \\
\hline$B \leftarrow 2 X_{P} X_{Q}$ & $F \leftarrow 2 X_{P} Z_{P}$ \\
\hline$C \leftarrow 2 Z_{P} Z_{Q}$ & $G \leftarrow X_{P}^{2}$ \\
\hline$D \leftarrow a_{4} A+a_{6} C$ & $H \leftarrow-4 a_{6} E$ \\
\hline$Z_{P+Q} \leftarrow A^{2}-B C$ & $I \leftarrow a_{4} E$ \\
\hline$X_{P+Q} \leftarrow B A+C D+2 x_{P-Q} Z_{P+Q}$ & $X_{2 P} \leftarrow F H+(G-I)^{2}$ \\
\hline & $Z_{2 P} \leftarrow 2 F(G+I)-E H$ \\
\hline
\end{tabular}

At the end of the scalar multiplication, an inversion is needed to recompute $x_{[k] G}=X_{[k] G} / Z_{k[G]}$. In our results this final inversion is taken in account, considering that we use little Fermat's theorem to compute the inversion (which is possible with our design, and does not cost any gate except in the sequencer).

The main feature of RNS compared to classical representation is that multiplication is almost free while all the computation complexity is on reduction. Therefore, it is interesting to find $A B+C D$ pattern in the addition and doubling law of the curve. This is done by the table given above in 13 reductions for 1 Montgomery ladder step (1 point-addition and 1 point doubling).

\subsection{Base Choice}

Our purpose is to use Kawamura's base extension in a massively parallel architecture. A value $r$ is set as the word depth, and $B$ and $\tilde{B}$ are chosen to be pseudo-Mersenne values $m_{i}=2^{r}-\epsilon_{i}$, with $\epsilon_{i}<2^{q}$ and $q<r / 2$. $\tilde{B}$ is chosen exactly the same manner. Regarding addition and doubling formulæ, we can set $\alpha$ of algorithm 1 to 45 . Indeed, the maximum value we have to reduce is $Z_{2 P} \leftarrow 2 F(G+I)-E H$. As $F, G, H$ and $I$ are less than $3 p, 2 F(G+I)$ is at most $36 p^{2}$. Since we can not afford to set negative input in $\operatorname{Red}_{M o n t g}$, and we are unable to verify that $2 F(G+I)>E H$, we have to calculate $(3 p-E) H$ which is positive, and less than $9 p^{2}$. Therefore $M>45 p$. As it is shown in Radix-RNS transformation subsection, $2^{r}$ is set as the $m_{0}$ value. In order to spare gates in our design, $q$ has to be as small as possible. If the targeted technology is a Stratix family FPGA, we will use $18 \times 18$ or $36 \times 36$ multipliers. Here are the main features of chosen bases for the use of $18 \times 18$ and $36 \times 36$ multipliers $(r$ is the word size in bits, $n$ is the number of parallel rower modules and $q$ is the max size of the $\epsilon_{i}$ in bits) :

\begin{tabular}{|c||c|c|c|c|c||c|c|c|c|c|}
\hline curve & 160 & 192 & 256 & 384 & 512 & 160 & 192 & 256 & 384 & 512 \\
\hline$r$ & 17 & 18 & 18 & 18 & 18 & 34 & 33 & 33 & 36 & 35 \\
\hline$n$ & 10 & 11 & 15 & 22 & 29 & 5 & 6 & 8 & 12 & 15 \\
\hline$q$ & 7 & 7 & 8 & 8 & 9 & 5 & 6 & 6 & 7 & 8 \\
\hline
\end{tabular}




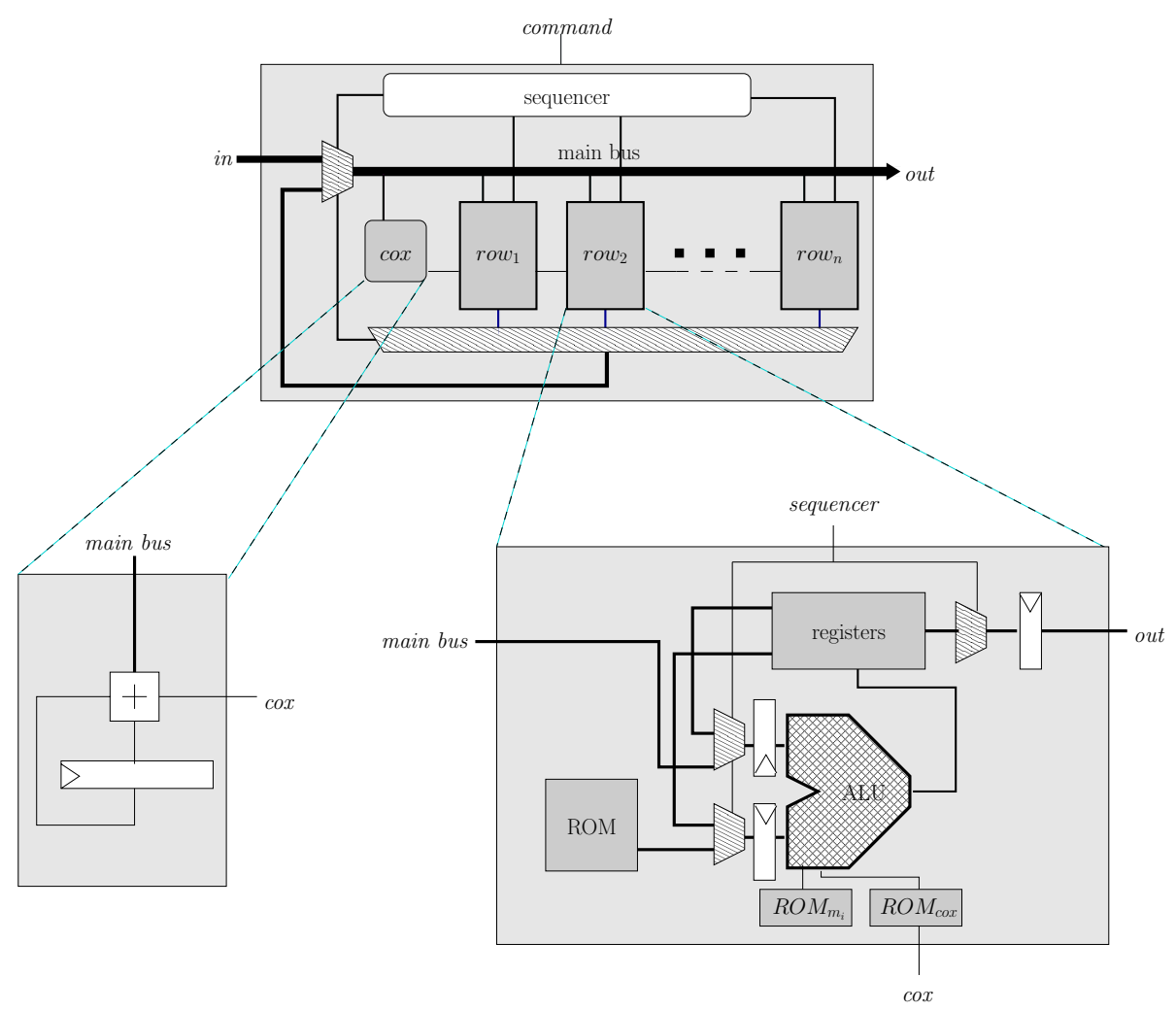

Fig. 1. General architecture and focus on the Cox and Rower design

\section{Hardware Architecture}

\subsection{Architecture Overview}

Already published architecture using RNS : Kawamura [10] proposed an architecture suitable for RSA calculation using his base extension algorithm. His general architecture is the same as ours and is given by the upper outline of the figure 1, He divided his design in multiple "Rower" modules, which were in charge of calculating $\left|\sum_{i=1}^{n}\left(M \xi_{i}\right) / m_{i}\right|_{m_{j}}$. and a "Cox" module in charge of calculating $\left\lfloor\sum_{i=1}^{n}\left(\xi_{i} / 2^{r}\right)\right\rfloor$. "Cox" design is very simple (a small adder). In [16] an improvement took the advantage of setting one cox per Rower. He then spared one cycle per reduction, computing $\gamma$ in the same cycle. The results were interesting, but the Rower pipeline was not deep enough to reach high clock frequency (3 stages).

Improvements in our design : Our architecture is an improvement of Kawamura's [10] [16] which makes it 
- suitable for elliptic curves,

- able to provide protections against side-channel attacks,

- designed to reach high clock frequencies.

The first limitation of Kawamura's architecture is the usage of only one RAM per channel. It only can execute a squaring, or multiply by a ROM data. This is not a limitation for RSA, the exponentiation algorithm only executes a square and a multiply by a constant, and so does the base reduction. This limitation is no more acceptable for elliptic curves. A general purpose register file (GPRs) must be added in order to multiply 2 local variables. Also the needed precalulation must be redefined. This point is focused in the subsection 3.3 .

The second limitation of Kawamura's architecture is the design of the pipeline core, which executes the operation $a c c=|x \times y+a c c|_{m_{i}}$. Kawamura's goal was to keep busy every pipeline stage $100 \%$ of the cycles. To do so, he designed a 3 stage pipeline, and needed to use 3 times less Rower than the number of channels he had in $B$ and $\tilde{B}$. Our architecture increases the pipeline depth to reach higher clock frequencies. We show that a $100 \%$ pipeline occupation is not really necessary for elliptic curves : it is easy to keep a good pipeline occupation even with deeper pipelines, with as many Rower as channels in $B$ and $\tilde{B}$, and with less channels for elliptic curves than for RSA (considering 160 bits curves versus 1024 bits bases for RSA). This point is focused in the subsection 3.2

Our architecture is showed on figure [1, It is the same as in [10] for the upper scheme part. Thus the Rower design (lower part of the scheme) is completely different. It is mainly composed with $n$ parallel channels which execute $a c c=$ $|x \times y+a c c|_{m_{i}}$ at each cycle and get operands and put the result from/in one of the 16 General Purpose Registers (GPR). Therefore, our architecture is able to compute a multiplication in $\mathbb{Z} / M \mathbb{Z}$ at each clock cycle. Our Rower architecture is described in 3.2

We propose a RNS-Radix transformation in subsection 3.4, and eventually discuss about consequences of our choice for resistance against side channel attacks in 3.5 .

\subsection{Pipeline Architecture}

In order to get high speed, the Arithmetical and Logical Unit(ALU) must execute $\left|r_{1} \times r_{2}\right|_{m}$ and accumulate the result at each clock cycle.

Considering this constraint, algorithm 3 computes the modular multiplication for any pseudo-Mersenne number. The accumulation may be executed at every step of the algorithm. For every value $P, P_{l s b}$ are the $r$ less significant bits of $P$, while $P_{m s b}=\left\lfloor P / 2^{r}\right\rfloor$.

As it is shown in algorithm 3 , there are in the proposed pipeline structure only 5 generic operands:

- a $r \times r$ multiplier,

- a $r \times q$ multiplier,

- a $q \times q$ multiplier, 

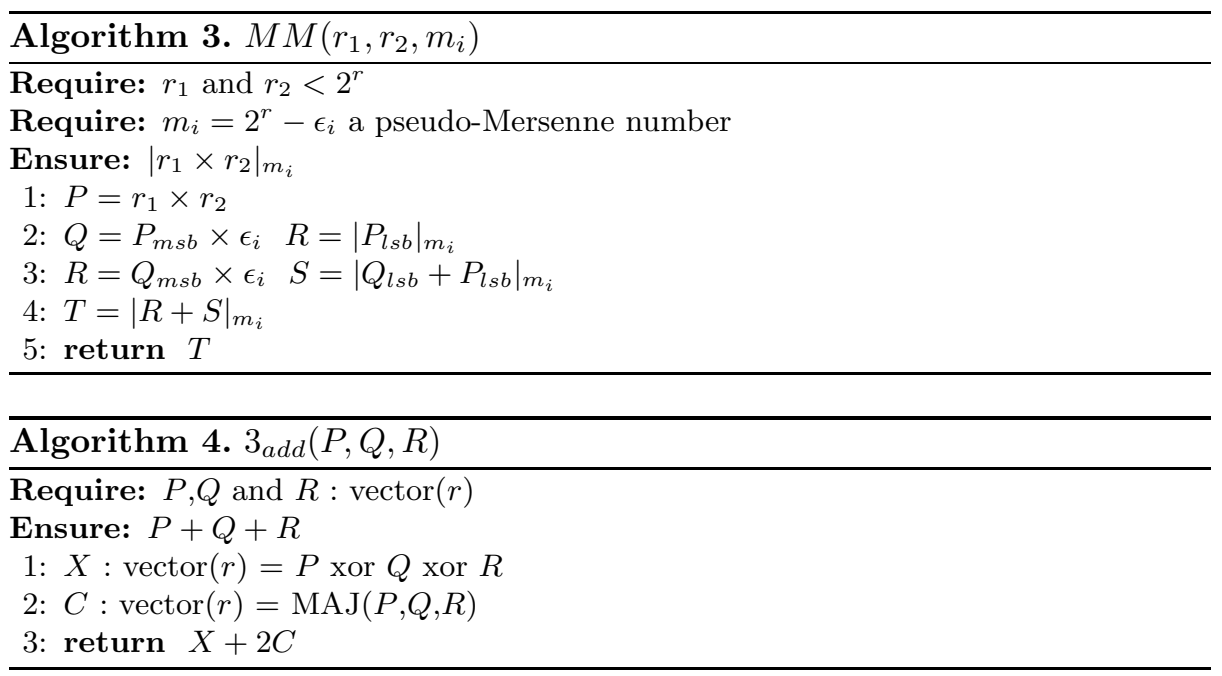

- $2 r$ modulo-adder taking two entries less than $m_{i}$, (4 if we consider accumulation, see the pipeline subsection)

- a $r$ modulo-adder taking one over two entries less than $m_{i}$, the other being less than $2^{r}$.

The multipliers are not an issue for FPGA, since both Altera Stratix and Xilinx Virtex families have got multiplier blocks. The following assumption are taken :

- A $a \times b$ multiplier will be implemented in a single FPGA DSP block, for every $a$ and $b<36$, even if $b$ is 9 bit wide.

- A $a \times b$ multiplier will be implemented in a single $9 \times 9$ blocks if both $a$ and $b$ are smaller than 9 .

These facts have been verified for every synthesis during this study.

Modular addition takes advantage on the fact that an addition by three operands of size $r$ only costs one LUT pass through and one addition of $r+1$ operands, by using algorithm 4 . Then, if $a$ and $b$ are less than $m_{i},|a+b|_{m_{i}}$ can be computed by computing in parallel $r_{1}=a+b$ and $r_{2}=a+b+\epsilon_{i}$ and by considering the carry of $r_{2}$ to choose the correct result. Figure 2 describes the adder design. If $m_{i}<a \leq 2^{r}$, an extra addition $a+b+2 \epsilon_{i}$ is required.

Two pipeline architectures are proposed in this paper (figure 21). Both try to balance the pipeline stages with one another, but make different assumptions :

- The first one makes the assumption that the a modular addition is twice faster than a multiplication.

- The second one makes the assumption that a modular addition runs as fast as multiplication.

To increase the pipeline occupation, we overlap independent operations : for example, the computation of $B$ and $C$ start before the one of $A$ is finished. Then, 

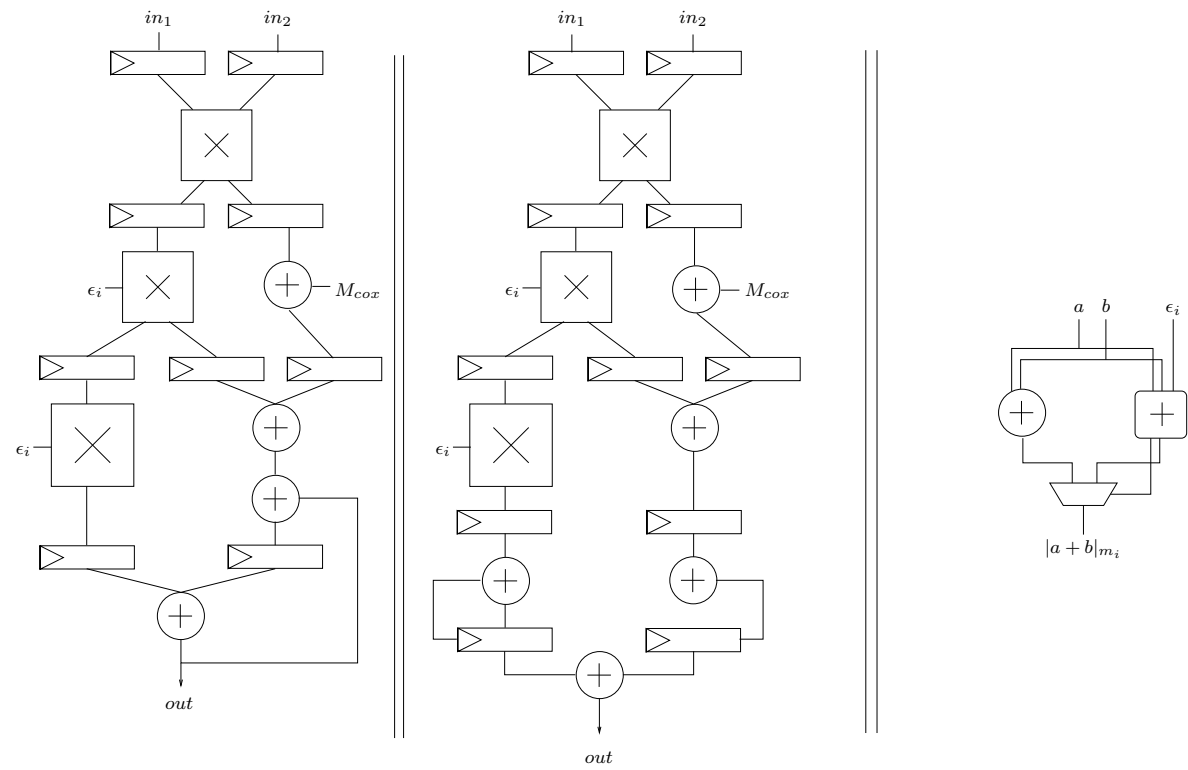

Fig. 2. 5 and 6 stage pipeline, and an adder modulo $m_{i}$

the wait states of the calculation of $A$, are taken up by $B$ and $C$. This technique increases the pipeline occupation, but may increase the number of needed general purpose registers. This is a trade-off. It is analysed in the subsection 3.3 .

On an Altera Stratix II chip, the maximum clock frequency of the 5 stage pipeline is $110 \mathrm{MHz}$, while the 6 stage reaches $158 \mathrm{MHz}$. At is is shown in subsection 3.3 , the percentage of idle states is respectively $95 \%$ and $90 \%$ for a pipeline of depth 5 and 6 , and a design with 5 channels (for a 160 bit curve with a channel length of 33 bits). This is the worst case of this study for pipeline occupation. We can then conclude that the 6 stage pipeline is the best choice for Stratix II technology. This study has to be done again for each target technology (including ASICs).

\subsection{Memory}

Precalculations and ROM content : The Rower main ROM is filled with the precalculated values described in this subsection.

Not considering reduction, we need during the calculation the following 3 variables : $a_{4}, a_{6}$ and $3 p: a_{4}$ and $a_{6}$ to compute $D$ and $I, 3 p$ to compute any subtraction (for example $Z_{P+Q}$ and $Z_{2 P}$ ). To compute $H$ it is possible to precompute $-4 a_{6}$. Eventually we need $\left|M^{2}\right|_{p}$ to compute Montgomery representatives. For computation we also need 0,1 and -1 for every channel. For radix-RNS transformation we need $2^{r}$ and for RNS-radix we need $2^{-r}$ and $-2^{-r}$ only on $\tilde{B}$ (see subsection 3.4). 


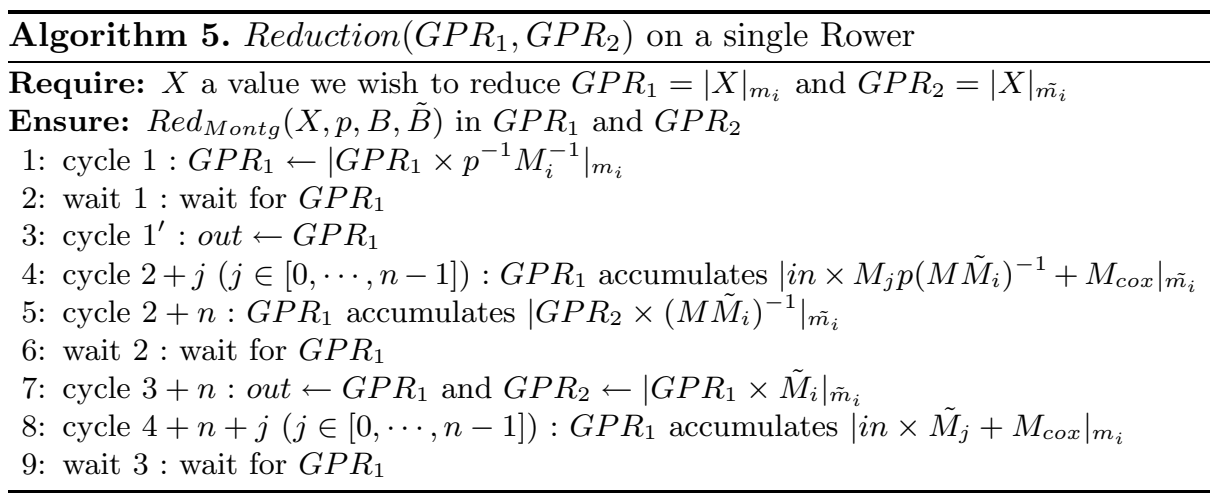

Algorithm 5 gives a fast version of the algorithm in our architecture. Cycle $1^{\prime}$ can be executed with another instruction. During cycles $2+j$ and $4+n+j$, the main bus is set to out $[j]$ the output of the $j^{\text {th }}$ channel. The needed precomputed values are

- for a channel $m_{i}$ of $B$ (modulo $\left.m_{i}\right)$ : $p^{-1} M_{i}^{-1}, \tilde{M}_{j}$ for $j \in[0 ; \cdots ; n-1]$.

- for a channel $\tilde{m}_{i}$ of $\tilde{B}$ (modulo $\tilde{m}_{i}$ ) : $M_{j} p\left(M \tilde{M}_{i}\right)^{-1}$ for $j \in[0 ; \cdots ; n-1],\left(M \tilde{M}_{i}\right)^{-1}$.

For a FPGA implementation, all these values may be set in ROMs, a curve change can be done by loading a different bitstream. Our results are given for fixed ROM. If it is necessary to change the curve during runtime, or if an ASIC implementation is needed, user may choose between 2 options :

- use RAMs instead of ROMs. This allows to change bases too, but all the values above have to be computed.

- reduce the number of curve-dependant values. Only 5 precomputed values per channel are needed : $p, a_{4}, a_{6},\left|M^{2}\right|_{p}$ and $\left|-p^{-1}\right|_{M}$. It costs 2 extra cycles and 2 extra wait per reduction.

For each Rower design, two extra small ROMs are needed, each one containing two values. The $R O M_{m_{i}}$ holds $m_{i}$ and $\tilde{m}_{i}$. The $R O M_{c o x}$ holds the value $\left.M p \tilde{M}_{i}{ }^{-1}\right|_{\tilde{m}_{i}}$ and $|-\tilde{M}|_{m_{i}}$ when the cox module set up the signal cox, these values are injected in the pipeline.

General purpose register file : Elliptic curves formulæ use local variables which have to be multiplied with one another, contrary to RSA which only has to square or multiply by a constant. That is why our architecture uses for every channel a general purpose register file (GPRs). Since every local variable has to be evaluated in $M$ and $\tilde{M}$, one need twice as GPR as the maximum of local variables.

As it is explained in the pipeline subsection, operations are overlapped : intermediate result computation may start before the previous is finished. This may implicate an increase of the number of needed registers. 
The following table shows that 16 GPR are enough : local variables are limited to 7 and reductions are at least executed by 2 , most of time by 3 . An 8 th local variable is taken by $x_{G}$ the exponentiated point abscissa. This leads to a pipeline fill rate of $90 \%$ for the 6 stage pipeline and 16 GPR per channels. This is a good trade-off.

\begin{tabular}{|c|c|c|}
\hline step & calculation & living variables \\
\hline 1 & $A B C$ & $X_{P} Z_{P} A B C$ \\
\hline 2 & $D Z_{P+Q}$ & $X_{P} Z_{P} A B C D Z_{P+Q}$ \\
\hline 3 & $X_{P+Q} E F$ & $X_{P} E F Z_{P+Q} X_{P+Q}$ \\
\hline 4 & $G H I$ & $E F G H I Z_{P+Q} X_{P+Q}$ \\
\hline 5 & $X_{2 P} Z_{2 P}$ & $X_{2 P} Z_{2 P} Z_{P+Q} X_{P+Q}$ \\
\hline
\end{tabular}

\subsection{Radix-RNS Transformation}

The RNS representation is not practical for using outside the design. Moreover, there is no need for extra material either to transform a number $X$ from its classical multiprecision representation $\left(X_{n-1}, \cdots, X_{0}\right)$ where $\left.X=\sum_{i=0}^{n-1} X_{i} 2^{i}\right)$ to its RNS representation $\left(x_{1}, \ldots x_{n}\right)$, nor the contrary. Using our architecture, the Radix-RNS transformation is trivial, if the sequencer can set up the main bus to the $X_{i}$ values, and $\left|2^{r}\right|_{m_{i}}$ is in ROM.

For RNS-Radix transformation, the main idea is to set the channel $m_{0}$ of $B$ to $2^{r}$. If it is so, $X_{0}=x_{0}$. To find $X_{1}$, all we need to do is to compute $X \leftarrow\left(X-x_{0}\right) / 2^{r}$. As $2^{r}$ is not co-prime with $M$, this computation is done over $\tilde{B}$, and the use of $B_{\text {ext }}(X, \tilde{B}, B)$ gives $X_{1} \leftarrow x_{0}$. By repeating this operation we compute $X_{2}, \cdots, X_{n}$.

This algorithm is not the most efficient but does not cost a single gate in our architecture. It never costs more than $0.3 \%$ of the total scalar multiplication time.

\subsection{Side Channel Attacks}

Our architecture supports an inherent capability to treat simple power analysis (SPA), or differential power analysis (DPA) and fault threats. Indeed, the Montgomery ladder is particularly efficient to counter both side channel attacks and fault attacks (no operation is dummy). Our finite state sequencer does not have any branch capability, bits of $k$ are only read at the beginning of each Montgomery-ladder step to invert registers. Therefore no information leaks from the computation time.

Moreover, randomness can be introduced at the very beginning of the algorithm by changing $G$ representation, replacing $\left(x_{G}, 1\right)$ by $\left(x_{G} \times a_{1}, a_{1}\right)$ and $\mathcal{O}=\left(a_{2}, 0\right)$, where $a_{1}$ and $a_{2}$ are random values. This countermeasure avoids Fouque's attacks [6] on collisions. The only SPA vulnerability is address-bit SPA attack [12, which is difficult to realize on real design. Moreover, to be DPA resistant $k$ may be added with $a_{3} \times \# C_{p, a_{4}, a_{6}}$ where $a_{3}$ is a random value. The impact on speed is $\log \left(a_{3}\right) / \log (p)$ on the speed. There is no impact on the design size. More robust randomizations of $k$ are also possible. 


\section{Result and Comparison}

In this section we give the overall results, and compare it to different architecture given in the open literature.

\subsection{Results}

Our target technology is the Altera Stratix family. This choice has very few impact on results compared to Xilinx Virtex family [18. We chose Altera because of the availability of the Quartus toolchain during the study. Among all the Altera products, we focus on 2 generations. First Stratix are the Altera FPGA at the $130 \mathrm{~nm}$ process node. The Xilinx equivalent is the Virtex II-pro. We also have fit our design in the Stratix II generation FPGA (the $90 \mathrm{~nm}$ process node), much more efficient. The equivalent is the Virtex IV by Xilinx.

We randomly chose elliptic curves of the following size : 160, 192, 256, 384 and 512 bits. No restriction were given for $p, a_{4}$ and $a_{6}$ but to be a valid elliptic curve. The result given below does not depend on the effective choice of $p$ but only on $\log _{2}(p)$. The number of Rower $n$ as well as the word depth $r$ are also given. These values are the most efficient considering the curve size.

For every fit we give the considered FPGA family and the exact reference of the chip. The maximum reachable frequency as well as the computation time for a whole scalar multiplication $[k] G$ are given. The size of the exponent $k$ is the same as the size of $p$. The final inversion, the $y_{[k] G}$ recalculation, the Radix-RNS and RNS-Radix transformations are included in the result.

Eventually the FPGA occupation is given. The Stratix FPGA are composed by logic elements (LE). LE are equivalent to the Look-Up Table (LUT) of the Virtex II-pro. Therefore a Virtex II-pro slice counts for two LE. No equivalent to the slice exists in the Stratix family. On the Stratix II family Altera introduced the Altera Logic Module (ALM), the Virtex IV slice equivalent. The number of used DSP blocks (multipliers) is also given. Altera gives the DSP occupation in numbers of $9 \times 9$ multipliers used. Stratix and Stratix II DSP blocks can indeed be configured as one $36 \times 36$ multiplier, two $18 \times 18$ or eight $9 \times 9$ multipliers.

\begin{tabular}{|c|c|c|c|c|c|c|c|c|}
\hline Family & curve & model & $n$ & $r$ & size & DSP & frequency & speed \\
\hline Stratix & 160 & EP1S20F484C5 & 5 & 34 & $11431 \mathrm{LE}$ & 74 & 92.6 & $0.57 \mathrm{~ms}$ \\
& 192 & EP1S30F780C5 & 6 & 33 & $12480 \mathrm{LE}$ & 80 & 89.6 & $0.72 \mathrm{~ms}$ \\
& 256 & EP1S60F780C5 & 8 & 33 & $16200 \mathrm{LE}$ & 125 & 90.7 & $1.17 \mathrm{~ms}$ \\
& 384 & EP1S80F1020C5 & 11 & 36 & $25279 \mathrm{LE}$ & 176 & 90.0 & $2.25 \mathrm{~ms}$ \\
& 512 & EP1S80F1020C5 & 15 & 35 & $48305 \mathrm{LE}$ & 176 & 79.6 & $4.03 \mathrm{~ms}$ \\
\hline Stratix II & 160 & EP2S30F484C3 & 5 & 34 & 5896 ALM & 74 & 165.5 & $0.32 \mathrm{~ms}$ \\
& 192 & EP2S30F484C3 & 6 & 33 & 6203 ALM & 92 & 160.5 & $0.44 \mathrm{~ms}$ \\
& 256 & EP2S30F484C3 & 8 & 33 & 9177 ALM & 96 & 157.2 & $0.68 \mathrm{~ms}$ \\
& 384 & EP2S60F484C3 & 11 & 36 & 12958 ALM & 177 & 150.9 & $1.35 \mathrm{~ms}$ \\
& 512 & EP2S60F484C3 & 15 & 35 & 17017 ALM & 244 & 144.97 & $2.23 \mathrm{~ms}$ \\
\hline
\end{tabular}

${ }^{1}$ The EP1S80 does not have enough DSP blocks, multipliers are fitted in the LE blocks, and frequency falls. 
The results given above show some properties of the chosen design. First the maximum frequency hardly falls with the size of the design. Indeed, no carry is propagated on the whole size of the operands due to RNS. The critical path is then not related to the design structure. For some fit it is in the sequencer, and for some other in a Rower. No further instruction has been given to the fitter except the pursuit of the maximal frequency.

As speed was our main concern, no RAM blocks were used. If size is a matter, ROMs and GPR may be fitted in RAM blocks, to spare logic. The Stratix family lacks some DSP for 512 bit curves, while Stratix II have far enough resources to fit for any cryptographic size (the EP2S60F484C3 is a middle size matrix in the Stratix 2 family).

\subsection{Comparison}

In this subsection we compare our design with other papers in the open literature. Our architecture supports any elliptic curve over $\mathbb{F}_{p}$ and is resistant to side channel attacks. We compare it with 3 papers we consider significant:

- the first one is another design based on RNS. In this paper, modular multiplication is realized through an Horner scheme. To our knowledge it is the only implementation using RNS for curves. It is realized by Schinianakis et al [23. It is implemented on an ASIC and on a FPGA.

- the second one is described in [1], and is based on a multi-precision algorithm. The main idea is an important work on the pipeline architecture for the classical multiprecision algorithm and on long word additions, through carry-look adder. It is realized on a Virtex II-pro. The scalar multiplication is based on a NAF recoding. This is to our knowledge the fastest implementation of elliptic curve scalar multiplication with generic curves. It outperforms every implementation given in [13] and is as fast as [21] using less resources.

- the third one is described in [7. The design is specific to a particular curve, $p$ being a pseudo Mersenne value. The main idea is a dual clock design, according DSP blocks to run at their maximum speed in Virtex 4 design, $500 \mathrm{MHz}$. It is the fastest FPGA implementation of elliptic curve scalar multiplication over $\mathbb{F}_{p}$, but with restrictions on $p$.

\begin{tabular}{|c|c|c|c|c|c|c|}
\hline paper & curve & FPGA family & FPGA model & size & freq.(MHz) & speed \\
\hline This work & 160 any & Stratix & EP1S20F484C5 & $11431 \mathrm{LE}$ & 92.6 & $0.57 \mathrm{~ms}$ \\
& 256 any & Stratix & EP1S60F780C5 & $16200 \mathrm{LE}$ & 90.7 & $1.17 \mathrm{~ms}$ \\
& 160 any & Stratix II & EP2S30F484C3 & 6203 ALM & 165.5 & $0.32 \mathrm{~ms}$ \\
& 256 any & Stratix II & EP2S30F484C3 & 9177 ALM & 157.2 & $0.68 \mathrm{~ms}$ \\
\hline$[23]$ & 160 any & Virtex & XCV1000E-8 & 21000 LUT & 58 & $1.77 \mathrm{~ms}$ \\
& 256 any & Virtex & XCV1000E-8 & 36000 LUT & 39.7 & $3.95 \mathrm{~ms}$ \\
\hline$[11]$ & 160 any & Virtex II-pro & XC2VP30 & $2171 \mathrm{sl}$. & 72 & $1 \mathrm{~ms}$ \\
& 256 any & Virtex II-pro & XC2VP30 & $3529 \mathrm{sl}$. & 67 & $2.27 \mathrm{~ms}$ \\
\hline$[7$ & 224 NIST & Virtex 4 & XC4VFX12 & $1580 \mathrm{sl}$. & 487 & $0.36 \mathrm{~ms}$ \\
& 256 NIST & Virtex 4 & XC4VFX12 & $1715 \mathrm{sl}$. & 490 & $0.49 \mathrm{~ms}$ \\
\hline
\end{tabular}


As a conclusion, our implementation is largely faster and smaller than [23]. Architecture [1] has got a better time surface trade-off (the ratio is about 1.5 if we consider that a Virtex 2 pro slice is equal to 2 Stratix LE, slices having not correspondant in Stratix products). Nevertheless it does not compute $k[G]$ as fast as ours, even on comparable technologies. It is eventually not resistant against side channel attacks. An overhead is needed to be SPA resistant. Ours is natively SPA resistant.

Architecture described in [7] is faster and presents a better size-area tradeoff regarding to ours (assuming that a Stratix II ALM and a Virtex IV slice are equivalent, and only considering slices). Guneysu's work only computes $[k] P$ over NIST curves, using pseudo-mersenne $p$. We can consider that using pseudo mersenne primes reduces the time complexity by a factor between 2 and 1.68 if lazy reduction is used. Then we can see that our results is competitive in term of latency regarding to his for 224 bit, and becomes better for 256 bits. Moreover, Guneysu's clock speed $(500 \mathrm{MHz})$ is particularly high and may represent an obstacle for industrial integration, and for an ASIC implementation (making the multipliers work twice as fast as the rest of the design would be impossible). Of course it is difficult to realize a fair comparison at this point since the two designs do not target the same curves, but RNS is a competitive alternative for general $F_{p}$ curves, especially for high security levels.

\section{Conclusion}

In this paper is presented a hardware architecture realizing elliptic curve scalar multiplication over any curve in $\mathbb{F}_{p}$, which uses RNS representations to speed up the computation. RNS supports a wide parallelization capability for arithmetic in $\mathbb{F}_{p}$. The overhead given by the elementary operation $\left(|a \times b|_{m}\right)$ is well pipelineable, even with large pipelines (6 stages), and contrary to RSA, the inherent parallelism of elliptic curve operations allows to easily fill the pipeline. Capability to support high clock frequency does not fall with the curve size.

In our future work, we will study other technologies like ASICs (with the usage of RAMs instead of the actual ROMs, other curve shapes, or other operations in elliptic curve cryptography, like pairings.

Acknowledgement : We would like to thank the anonymous referees for their detailed review of this paper and their helpful suggestion. Thanks to David Lubicz, Sylvain Duquesne and Jeremie Detrey for their contribution to this work.

\section{References}

1. Bajard, J.-C., Didier, L.-S., Kornerup, P.: An rns montgomery modular multiplication algorithm. IEEE Transactions on Computers 47(7), 766-776 (1998)

2. Bajard, J.-C., Imbert, L., Liardet, P.-Y., Teglia, Y.: Leak resistant arithmetic. In: Joye, M., Quisquater, J.-J. (eds.) CHES 2004. LNCS, vol. 3156, pp. 116-145. Springer, Heidelberg (2004) 
3. Chen, L., Yanpu, C., Zhengzhong, B.: An implementation of fast algorithm for elliptic curve cryptosystem over GF(p). Journal of Electronics (China) 21(4), 346-352 (2004)

4. Coron, J.-S.: Resistance against differential power analysis for elliptic curve cryptosystems. In: Koç, Ç.K., Paar, C. (eds.) CHES 1999. LNCS, vol. 1717, pp. 292-302. Springer, Heidelberg (1999)

5. Edwards, H.: A normal form for elliptic curves. Bull. Amer. Math. Soc. 44 (2007)

6. Fouque, P.-A., Valette, F.: The doubling attack - why upwards is better than downwards. In: Walter, C.D., Koç, Ç.K., Paar, C. (eds.) CHES 2003. LNCS, vol. 2779, pp. 269-280. Springer, Heidelberg (2003)

7. Güneysu, T., Paar, C.: Ultra high performance ecc over nist primes on commercial fpgas. In: Oswald, E., Rohatgi, P. (eds.) CHES 2008. LNCS, vol. 5154, pp. 62-78. Springer, Heidelberg (2008)

8. Jarvinen, K.U., Skytta, J.O.: High-speed elliptic curve cryptography accelerator for koblitz curves. In: Annual IEEE Symposium on Field-Programmable Custom Computing Machines, pp. 109-118 (2008)

9. Joye, M., Sung-Min-Yen: The montgomery powering ladder. In: Kaliski Jr., B.S., Koç, Ç.K., Paar, C. (eds.) CHES 2002. LNCS, vol. 2523, pp. 291-302. Springer, Heidelberg (2003)

10. Kawamura, S., Koike, M., Sano, F., Shimbo, A.: Cox-rower architecture for fast parallel montgomery multiplication. In: Preneel, B. (ed.) EUROCRYPT 2000. LNCS, vol. 1807, pp. 523-538. Springer, Heidelberg (2000)

11. Mentens, N.: Secure and Efficient Coprocessor Design for Cryptographic Applications on FPGAs. PhD thesis, Ruhr-University Bochum (2007)

12. Messerges, T.S., Dabbish, E.A., Sloan, R.H.: Power analysis attacks of modular exponentiation in smartcards. In: Koç, Ç.K., Paar, C. (eds.) CHES 1999. LNCS, vol. 1717, pp. 144-157. Springer, Heidelberg (1999)

13. de Dormale, G.M., Quisquater, J.-J.: High-speed hardware implementations of elliptic curve cryptography: A survey. J. Syst. Archit. 53(2-3), 72-84 (2007)

14. Miller, V.S.: Use of elliptic curves in cryptography. In: Williams, H.C. (ed.) CRYPTO 1985. LNCS, vol. 218, pp. 417-426. Springer, Heidelberg (1986)

15. Montgomery, P.L.: Modular multiplication without trial division. Mathematics of Computation 44, 519-521 (1985)

16. Nozaki, H., Motoyama, M., Shimbo, A., Kawamura, S.-i.: Implementation of rsa algorithm based on rns montgomery multiplication. In: Koç, Ç.K., Naccache, D., Paar, C. (eds.) CHES 2001. LNCS, vol. 2162, pp. 364-376. Springer, Heidelberg (2001)

17. National Institute of Science and Technology. The digital signature standard. Technical report, http://csrc.nist.gov/publications/fips/archive/fips186-2/fips186-2.pdf

18. White Paper. Stratix vs. virtex-ii pro fpga performance analysis. Technical report, http://www.altera.com/literature/wp/wpstxvrtxII.pdf

19. Posch, K.C., Posch, R.: Modulo reduction in residue number systems. IEEE Trans. Parallel Distrib. Syst. 6(5), 449-454 (1995)

20. Ecegovac, M., Duquesne, S., Bajard, J.C.: Combining leak-resistant arithmetic for elliptic curves define over $\mathbb{F}_{p}$ and rns representation

21. Sakiyama, K., Mentens, N., Batina, L., Preneel, B., Verbauwhede, I.: Reconfigurable modular arithmetic logic unit for high-performance public-key cryptosystems. In: Bertels, K., Cardoso, J.M.P., Vassiliadis, S. (eds.) ARC 2006. LNCS, vol. 3985, pp. 347-357. Springer, Heidelberg (2006) 
22. Satoh, A., Takano, K.: A scalable dual-field elliptic curve cryptographic processor. IEEE Transactions on Computers 52, 449-460 (2003)

23. Schinianakis, D.M., Fournaris, A.P., Michail, H.E., Kakarountas, A.P., Stouraitis, T.: An rns implementation of an fpelliptic curve point multiplier. Trans. Cir. Sys. Part I 56(6), 1202-1213 (2009)

24. Shenoy, P.P., Kumaresan, R.: Fast base extension using a redundant modulus in rns. IEEE Trans. Comput. 38(2), 292-297 (1989)

25. Szerwinski, R., Gayneysu, T.: Exploiting the power of GPUs for asymmetric cryptography. In: Oswald, E., Rohatgi, P. (eds.) CHES 2008. LNCS, vol. 5154, pp. 79-99. Springer, Heidelberg (2008) 